La extensión en la formación del educador musical. Una experiencia de prácticas musicales situadas en el territorio Mauro Valicente

Extensión en red (N. ${ }^{\circ}$ 8), e004, septiembre 2017

ISSN 1852-9569 | http://perio.unlp.edu.ar/ojs/index.php/extensionenred

FPyCS | Universidad Nacional de La Plata

La Plata | Buenos Aires | Argentina

\title{
La extensión en la formación del educador musical Una experiencia de prácticas musicales situadas en el temitorio
}

\author{
The Extension in the Professional Education of the Music Educator \\ An Expenience of Musical Practic es Loc ated in the Temitory
}

\author{
Mauro Valicente \\ maurovalicente@gmail.com \\ https://orcid.org/0000-0003-3975-0346 \\ Laboratorio para el Estudio de la Experiencia Music al (LEEM) \\ Facultad de Bellas Artes \\ Universidad Nacional de La Plata | Argentina
}

\section{Resumen}

El autor reflexiona en este escrito sobre el rol de la actividad extensionista como parte de la formación profesional, a partir del trabajo desarrollado en el marco del proyecto «Co-creando espacios de comunicación mediante la música. Una propuesta situada de musicalidad comunicativa en el territorio», radicado en la Facultad de Bellas Artes (FBA) de la Universidad Nacional de La Plata (UNLP).

\section{Palabras clave}

extensión universitaria, formación profesional, prácticas musicales, territorio

\section{Abstract}

In this paper the author reflects about the role of the extensionist activity as part of the professional training, based on the work developed within the framework of the project «Co-Creating Spaces of Communication through Music. A Proposal of Communicative Musicality in the Territory», located in the Faculty of Bellas Artes (FBA) of the National University of La Plata (UNLP).

\section{Keywords}

university extension, vocational training, musical practices, territory 


\section{La Extensión como propósito de la Universidad Nacional de La Plata}

La extensión universitaria es una de las seis líneas estratégicas planteadas en el Plan estratégico. Gestión 2014-2018 (2014) de la Universidad Nacional de La Plata y tiene como fin «promover la utilidad social del conocimiento y la práctica solidaria y formativa, contribuyendo a la mejora de la calidad de vida de la población, la reconstrucción del tejido social, el desarrollo sustentable y el fortalecimiento de la identidad cultural» (p. 20). Asimismo, se propone como objetivo general el desarrollo de prácticas que promuevan la articulación, la comunicación y el intercambio de saberes que contribuyan a la resolución de diferentes problemáticas sociales, desde una perspectiva integral e interdisciplinaria, integrando a la universidad con la comunidad de la cual forma parte.

Se considera que la inserción de la extensión a la currícula universitaria contribuye a la formación y a la capacitación de estudiantes, de graduados y de profesionales con una visión integral de sus disciplinas y un compromiso social. Del mismo modo, se afirma que la integración de estas acciones extensionistas a la actividad docente y de investigación -junto con la promoción y la cooperación del trabajo interdisciplinario- favorece la construcción de respuestas dirigidas a la comunidad, propiciando espacios de participación y de expresión en distintas instancias, y fomentando la construcción de identidad.

En el análisis de las fortalezas, las oportunidades, las debilidades y las amenazas (FODA) que se describen en el Plan (2014, p. 55) se detalla la relación con la actividad de extensión. En cuanto a las primeras, se hace referencia, por ejemplo, a la fuerte promoción, al incremento de actividades extensionistas y a la consolidación de los Centros Comunitarios de Extensión (CCEU) como lugar de referencia local y del Consejo Social que convoca a distintos representantes y sectores de la comunidad. Respecto de las oportunidades, se menciona la jerarquización a nivel nacional de políticas extensionistas y las importantes convocatorias externas a la universidad que permiten el desarrollo de proyectos y de programas, entre otros. En cuanto a las debilidades y a las amenazas se detalla la falta de material bibliográfico junto con los escasos recursos económicos destinados a financiar proyectos de extensión, la falta de sistematización de las experiencias y de instrumentos metodológicos adecuados (que impiden medir el impacto de las acciones realizadas) y la falta de la incorporación curricular de la extensión universitaria, lo que podría generar una falta de motivación y una inclinación de los docentes por cumplir con otras tareas que no impliquen a esta función de la universidad como prioridad. 
El escenario actual, según el documento de la UNLP, es de una compleja situación social que atraviesa la comunidad y que presenta demandas que exigen profesionales capaces de responder, de intervenir y de articular sus saberes con los del territorio, así como también el compromiso de la universidad en participar en los diseños de las políticas públicas. Algunos temas, como el trabajo con las comunidades en condiciones de vulnerabilidad, la defensa de los derechos humanos, la construcción de identidad y las violencias expresadas en distintos niveles, demandan la actualización y la reflexión sobre el rol profesional, así como la intervención desde un ámbito público con un mecanismo que atienda a la resolución de las distintas problemáticas de la actualidad.

A partir del proceso de jerarquización y de crecimiento constante que atraviesa la extensión universitaria, favorecida por una fuerte política de gestión en los últimos años, es necesario abordar un desafío mayor, que implica estar a la altura de estas demandas sociales, contribuyendo a mejorar la calidad de vida de la población, a la recontracción del tejido social y al fortalecimiento de la identidad.

\section{La experiencia extensionista}

El proyecto que enmarca este escrito se denomina «Co-creando espacios de comunicación mediante la música. Una propuesta situada de musicalidad comunicativa en el territorio» y se desarrolló durante 2016 como parte de las actividades llevadas a cabo por un grupo de docentes investigadores pertenecientes al Laboratorio para el Estudio de la Experiencia Musical (LEEM) de la Facultad de Bellas Artes (UNLP).

Los fundamentos que dieron origen al proyecto provinieron de dos vertientes: por un lado, los conocimientos surgidos de la investigación en la Psicología Cognitiva y Social de la Música y la Educación Musical; por otro, de la labor realizada previamente por uno de los miembros del equipo en tres ONG de La Plata (Buenos Aires, Argentina) y sus alrededores, lo que permitió contextualizar las actividades en la realidad de cada territorio.

El proyecto se propuso propiciar un encuentro con la comunidad por medio de la implementación de modalidades de práctica musical, fundamentadas en los aspectos intersubjetivos inherentes a la musicalidad comunicativa (Malloch \& Trevarthen, 2009). Bajo este concepto, se gestan durante la práctica musical expresiones compartidas cuyas pautas temporales, dinámicas, espaciales y narrativas ponen en comunión rasgos 
idiosincráticos y de la cultura. Desde esta perspectiva, se privilegia la contextualización de la práctica musical en el territorio, entendiendo a la práctica expresiva situada como el emergente co-creado por los participantes en dicho espacio.

El objetivo que guió la realización del proyecto fue el de desarrollar nuevas modalidades para el abordaje de prácticas musicales basadas en la participación, lo que implica la construcción cooperativa intersubjetiva entre adultos y niños/as o entre niños/as y orientada a generar narrativas musicales de expresión y de intención, de modo de elaborar mediante la participación un sentimiento compartido del tiempo. La realización de actividades expresivas que ponen en juego rasgos de musicalidad comunicativa en contextos de práctica musical espontánea tiene gran relevancia, en tanto cumple una función vertebradora de actuaciones expresivas que son frecuentemente limitadas o censuradas debido a prejuicios socioculturales muy instalados, vinculados a las concepciones del talento en el arte y su supuesta distribución desigual en la población. A su vez, la realización contribuye a configurar entornos propicios para llevar adelante modos sociocomunicativos de validez ecológica en el medio comunitario para los actores comprometidos.

Las instituciones comunitarias en las que se desarrollaron las actividades son tres ONG insertas en contextos desfavorecidos:

- Hogar Chispita. Obra del Padre Cajade. Barrio Los Hornos (La Plata, Buenos Aires).

- Los Gurises. Copa de leche y talleres. Barrio Melchor Romero (La Plata, Buenos Aires).

- Biblioteca« Del otro lado del Árbol». Parque Saavedra (La Plata, Buenos Aires).

Se formaron tres grupos de trabajo, uno para cada institución. La actividad consistió en la realización de prácticas musicales, con formato taller, de una hora semanal. No obstante, debido a las características propias de cada institución, que resultan en contextos diferentes, cada uno diseñó y desarrolló actividades de diversa índole, situadas en la realidad de cada territorio.

Mi experiencia como extensionista tuvo lugar en el Hogar Chispita. Éste se encuentra en las periferias de la ciudad de La Plata y realiza diferentes tareas de asistencia, de contención y de apoyo a niños/as de distintas edades en situaciones de precariedad y con diferentes problemáticas. 
El trabajo en la institución se realizó con un grupo de niños/as cuya franja etaria estuvo comprendida entre los seis y los once años. El número de integrantes fue variando a lo largo del año en virtud de la asistencia o de la inasistencia. La propuesta pedagógicodidáctica del proyecto estuvo enmarcada en torno a los rasgos de la musicalidad comunicativa, privilegiando la comunicación musical en las diferentes actividades.

La metodología de las clases se orientó hacia la realización de prácticas participativas desarrolladas a partir de un repertorio variado de canciones, de juegos y de rimas (propuestas tanto por los docentes como por los alumnos) y la ejecución de instrumentos, tanto de forma grupal como individual. La ejecución instrumental fue uno de los objetivos centrales de cada actividad, ya que uno de los focos de interés estuvo puesto en las características de la interacción que se suscita entre los participantes durante el hacer musical. En ciertos momentos, se dio lugar a la improvisación, en la que los alumnos proponían diferentes ritmos ejecutados sobre una base musical interpretada por el docente. En varias ocasiones, y a pedido de los chicos, se debieron repetir algunas actividades que eran de su preferencia. Cuando comenzaron a desarrollarse las actividades, se indagó acerca de sus gustos musicales y de sus inquietudes, con el fin de incorporar estos datos a la práctica de modo que resultara más motivador para el grupo. En todo momento se trabajaron, de forma paralela, aspectos de la comunicación dentro del grupo, así como el respeto y el valor de la clase de música.

La evaluación permanente de estas actividades permitió aislar una serie de aspectos que dificultaron la ejecución del proyecto, entre ellos, la ya mencionada modificación en el número de chicos en función de las asistencias o las inasistencias a las clases, lo que incidió en la continuidad del trabajo. Dicha continuidad también se vio afectada por circunstancias climáticas ya que, en días de lluvia, se debieron suspender clases por las condiciones edilicias del lugar. Asimismo, las diversas problemáticas cotidianas de los alumnos (falta de contención familiar, cuestiones culturales y de género, etc.), incidieron inicialmente en el establecimiento de un vínculo entre los chicos y los docentes, lo cual complejizó la indagación acerca de los aspectos de la musicalidad comunicativa.

Las actividades realizadas en las clases buscaron la participación de los alumnos, ya sea mediante el aporte de sus propias inquietudes o de la propuesta de los responsables extensionistas, pero esto se logró sólo en algunos momentos debido a las diversas problemáticas presentes, que se evidenciaban en situaciones como el déficit de la atención o la falta de interés. Por ello, se puso el énfasis en inculcar formas de comunicación, de convivencia y de comportamiento (entre los chicos/as o entre ellos y los docentes) en 
varias clases, lo que desvió momentáneamente el objetivo inicial. Si bien se logró modificar los hábitos a lo largo de los encuentros, esto se vio obstaculizado en virtud del tiempo transcurrido entre cada clase.

No obstante, también pudieron observarse una serie de aspectos positivos y de fortalezas. El vínculo retroalimentador que buscó establecer el proyecto entre la actividad académica y los sectores beneficiarios posibilitó que los chicos de la institución accedieran a actividades musicales, algo que, de otro modo, les hubiese resultado inaccesible. Este contacto incentivó a algunos de ellos a manifestar interés por continuar con algún tipo de estudio musical, por ejemplo, aprender a «tocar la guitarra». Además, el carácter situado de las prácticas permitió conocer y tener en cuenta las preferencias de los grupos, lo cual promovió un grado de compromiso con la actividad por parte de varios de ellos. Es de destacar la buena predisposición y el acompañamiento de las personas a cargo de la ONG, ya que, a pesar de las problemáticas existentes en el territorio, el escaso presupuesto y/o los inconvenientes ocasionados por distintos factores - como las inclemencias climáticas-, en todo momento fueron partícipes directos de las actividades de música demostraron entusiasmo e interés, aportaron ideas y colaboraron con los docentes en la contención del grupo.

\section{Aportes de la actividad extensionista para la formación profesional}

Participar en el proyecto de extensión constituyó una experiencia enriquecedora para todos los integrantes del equipo. En términos generales, se considera que esta actividad constituye una experiencia de aprendizaje constante, que permite poner en práctica los conocimientos adquiridos durante los años de estudio, en un contexto de actuación real. En cuanto al tema específico, dio lugar a conocer y a abordar una modalidad de trabajo diferente a la habitual en las prácticas de enseñanza de la música. Esto significó reemplazar la concepción tradicional de la clase de música que ha regido durante mucho tiempo en contextos áulicos por una mirada más actual que propicia la práctica musical compartida y la participación activa de los alumnos por medio de actuaciones expresivas espontáneas, todas tenidas en cuenta en la co-creación de narrativas musicales que dan lugar a un sentimiento compartido del tiempo. Esto posibilitó entender ciertos rasgos de la musicalidad comunicativa, como ser la comunicación intersubjetiva en la ejecución grupal. 
Si bien las condiciones dificultaron —en alguna medida - el desarrollo de las actividades tal como habían sido previstas, el compromiso de todos los miembros del equipo extensionista promovió la búsqueda constante de alternativas en pos de mejorar las prácticas y el manejo de los grupos. El desafío de llevar a cabo actividades sostenidas en un contexto desfavorecido, en el cual los alumnos afrontan diversas problemáticas, hizo que, en varias ocasiones, se tuviera que replantear los modos de abordar las actividades.

El contexto actual en el que se encuentra el Hogar y la realidad del sector social que atiende permitió advertir la importancia y el papel fundamental de la extensión universitaria en dichos contextos, acordando con Gabriela Loustanau y Andrea Rivero en que la extensión «debiera contribuir a la transformación de la realidad aportando a la resolución de problemáticas sociales complejas» (2016, p. 39). A partir de la experiencia realizada, se considera que sería muy valioso continuar promoviendo el desarrollo de este tipo de proyectos, así como la participación de los estudiantes en ellos.

\section{Reflexiones finales}

En el marco del proyecto, se propuso privilegiar la contextualización de la práctica musical en el territorio, entendiendo a la práctica expresiva situada como el emergente co-creado por los participantes en dicho espacio. Asimismo, se buscó establecer un vínculo retroalimentador entre la actividad académica y la realidad de los sectores beneficiarios, entendiendo que es a través de la puesta en acto donde los conocimientos desarrollados mediante la labor académica adquieren sentido.

Estas ideas encuentran vinculación con el concepto de «integralidad» planteado por Gabriel Kaplún (2012), quien la define como «un movimiento que, sin descuidar los aprendizajes y la producción de conocimiento, pone a la extensión en el centro de las funciones universitarias» (p. 40). Es decir, permite la retroalimentación entre los conocimientos producidos en la universidad y en la sociedad, para su transformación. En esos procesos, los universitarios «aprenden investigando e interviniendo y se transforman también ellos y la institución» (Kaplún, 2012, p. 37).

Sobre la base de la experiencia desarrollada, se considera que sería importante formalizar las prácticas extensionistas en los planes de estudio como una práctica permanente, organizada y orientada a lo largo de los años de la carrera y no como una actividad aislada. 
Esto aportaría a la formación integral del estudiante en el ámbito académico, tanto por medio de la articulación con la docencia y con la investigación, como por la posibilidad de situar los conocimientos en el territorio, produciendo así profesionales comprometidos socialmente ya que «son las prácticas las que permiten posicionar actores universitarios comprometidos con la realidad y la transformación social» (Loustaunau \& Rivero, 2016, p. 38). A su vez, contribuiría a la integralidad entre la universidad y la transformación de las complejas problemáticas que presenta la sociedad en la actualidad. Se cree importante asumir un enfoque crítico de las prácticas y de la formación, con el fin de despejar posibles obstáculos y de mejorar el trabajo de extensión.

\section{Referencias}

KAPLÚN, G. (2012). La integralidad como movimiento instituyente en la universidad. Intercambios, (1), s/p. Recuperado de http://intercambios.cse.edu.uy

LOUSTANAU, G. y RIVERO, A. (2016). Desafíos de la curricularización de la extensión universitaria. Masquedós, 1(1), 37-45.

MALLOCH, S. y TREVARTHEN, C. (2009). Communicative Musicality. Oxford, United Kingdom: Oxford University Press.

UNIVERSIDAD NACIONAL DE LA PLATA (2014). Plan estratégico de la UNLP. Gestión 2014-2018. La Plata, Argentina: Universidad Nacional de La Plata. Recuperado de http://www.unlp.edu.ar/uploads/docs/plan_estrategico_2014_2018.pdf 